\title{
SENSITIVITY AND STRENGTH: EFFECTS OF INSTRUCTIONS ON RESISTANCE TO CHANGE
}

\author{
CHRISTOPHER A. PODLESNIK and PHILIP N. CHASE \\ West Virginia University
}

\begin{abstract}
Several research laboratories have found that instructed behavior can be less sensitive to changes in contingencies than shaped behavior. The current experiment examined whether these differences in sensitivity could be related to resistance to change. Two groups of subjects, who were matched on the basis of an initial disruption assessment, were exposed to a variable-interval 30-s schedule of reinforcement with and without a disrupter. The disrupter was a video presentation of a popular television situation comedy. One group received minimal instructions (MI) that told them only that they could earn points exchangeable for money. Each member of the second group received a complete instruction (CI) that described the topography of the target response that was yoked to a MI subject's stable baseline response rate. The response rates under the disruption condition for the $\mathrm{Cl}$ subjects were more resistant to change than the $\mathrm{Ml}$ subjects in 14 out of 15 disruption sessions. These findings are discussed in terms of resistance to change being increased by instructional conditions like those manipulated and that the procedures used to test disruption provide an additional method to evaluate differences between instructed and contingency-governed behavior.
\end{abstract}

Rules, instructions, policies, and directions are assumed to influence behavior and are used to teach, inform, or prescribe behavior in many kinds of situations. How rules affect behavior, however, is still not clearly understood. For this reason, research on the effect of rules on behavior has been a major focus for a number of researchers. Skinner (1969) defined rules as contingency-specifying discriminative stimuli $\left(S^{D}\right)$ that describe behavior and the controlling environment. In defining rules, Skinner emphasized the differences between behavior that is influenced by direct contact with contingencies of reinforcement and behavior that is influenced by descriptions of those contingencies. According to Skinner, contingency-governed behavior is selected by direct contact with the consequences for the behavior in question whereas rule-governed 
behavior is maintained by rules and the contingencies supporting the following of rules. Skinner suggested that the controlling variables are different for contingency-governed and rule-governed behavior even when the behaviors are topographically similar.

\section{Sensitivity}

One of the primary issues concerning these differences between rulegoverned behavior and other behavior is that many studies have found that instructed behavior is insensitive to changes in contingencies. When instructed on one schedule (e.g., fixed-ratio), subjects will often continue to respond in accordance with instructions even though the contingency changes (e.g., to a variable-interval schedule) (Kaufman, Baron, \& Kopp, 1966). For example, Shimoff, Catania, and Matthews (1981) taught subjects to respond on either a tandem random-interval (RI) differential-reinforcement-of-low-rates (DRL) or a tandem random-ratio (RR) DRL with either instructions or by exposing the subjects to the contingencies. After training was complete, uninstructed subjects' behavior was sensitive to the removal of the DRL intervals in 10 out of 13 cases, whereas instructed subjects' behavior was sensitive in only 8 out of 20 cases. Shimoff et al. concluded that insensitivity to changes in contingencies was due to the instructions.

Although instructed behavior can be made sensitive to the changes in local contingencies of reinforcement (Galizio, 1979; Joyce \& Chase, 1990; LeFrancois, Chase, \& Joyce, 1988), the insensitivity of some kinds of instructed behavior is important for applied, methodological, and theoretical reasons. If the goal of teaching or training is to produce behavior that adapts to changes in consequences, then one should not assume that instructions will produce adaptive behavior. In fact some have implicated the relation between instructional control and rigidity (Wulfert, Greenway, Farkas, Hayes, \& Dougher, 1994). If instructions are used as part of the methods of an experiment, then one should not assume that the behavior achieved is the same as the behavior achieved without instructions (Galizio, 1979). At the theoretical level, these differences between instructed and noninstructed behavior suggest questions related to the contingencies operating on rule-governed behavior.

One such question is whether rule-governed behavior is typically at greater strength than the specific classes of responding to which it is compared. For example, one may account for the insensitivity of rule-governed behavior found in the typical experiment by referring to the strength of rule-governed behavior as a result of the extensive and generalized history the human subjects have had for following rules. In most cases, rule following has been reinforced with a range of different topographies of behavior and specific consequences. Given such typical histories, one should not be surprised to find that instructed behavior often tends to be less sensitive to changes in contingencies because it is stronger than the contingency-governed behavior to which it is compared. In current behavioral terms, differences in strength, may be described in terms of resistance to change. 


\section{Resistance to Change}

Nevin, Mandell, and Atak (1983) suggested that response strength is analogous to momentum in physics and can be described as behavioral momentum. In physics, the product of velocity and mass is described as momentum. The momentum of an object is the degree to which an opposing force will alter its velocity. By increasing an object's mass, one can proportionally increase that object's momentum. An outside force will decrease the velocity much less for a very dense object traveling at 100 $\mathrm{mph}$ than a less dense object traveling at the same speed. According to Nevin et al., response rate is analogous to velocity, behavioral disrupters (i.e., competition from other contingencies) are analogous to opposing forces, and rate of reinforcement is analogous to mass.

Continuing with this analogy, responses reinforced at a higher rate have been found to be more resistant to a disrupter than responses reinforced at a lower rate. For example, Mace et al. (1990) had mentally retarded adults sort different-colored plastic dinnerware in each component of a multiple schedule. When a video disrupter was introduced, the sorting rate for the color with a higher reinforcement rate decreased less than the sorting rate for the color with a lower reinforcement rate. Dube and Mcllvane (2001) studied prefeeding, response-independent food, and alternative tasks as disrupters in discrimination procedures with severely mentally retarded subjects. Differences in resistance to change increased with differences in rates of reinforcement between components. The more similar reinforcement rates were, the more equivalent the resistance to change of responding across components.

Given these findings from the behavioral momentum literature, it seems possible that another way to examine the insensitivity of rulegoverned behavior to changes in contingencies is by using the procedures of the behavioral-momentum literature to measure resistance to disruption. In order to extend our understanding of rules and their effects on behavior, it would help to determine how instructions affect behavior within experiments that use problems that test for resistance to change. The present study attempted to determine the effects of a visual disrupter on instructed versus contingency-governed responding on a VI schedule.

\section{Method}

\section{Subjects}

Six subjects recruited by the experimenter from introductory psychology classes at West Virginia University completed the present study for financial compensation. Eighteen subjects did not meet criteria for completing the experiment (described below). Subjects were informed that they could earn points exchangeable for money based on their performance. When subjects finished the experiment, they also received a $\$ 1.00$ bonus for each day of participation, which was paid at the end of the experiment along with the performance pay. Following each session, subjects received a slip of paper indicating how much they earned for 
that session. All subjects were asked to read and sign a Consent and Information Form prior to their participation in the experiment.

\title{
Settings and Apparatus
}

Subjects worked in a $2-\mathrm{m}^{2}$ room containing a chair, desk, the apparatus, and a $.3-\mathrm{m}^{2}$ door connected to the experimenter's room for communicating with the experimenter. An IBM compatible, Pentium II $450 \mathrm{MHz}$ computer sat atop the desk with an 18-in monitor at eye level. A Microsoft mouse was used in some phases of the research and an IBM 101-key keyboard was used for the remainder of the experiment. All keys on the keyboard were covered by a hard Plexiglas guard except the number pad and the escape key. The visual interface included a dragging task (described below under Disruption assessment) and simple arithmetic addition problems (described below under Instructional comparison) displayed on the computer screen. The visual interface of the computer and schedules of reinforcement that were used to provide points were programmed on the computer in Microsoft Visual Basic 6.0 for Windows. A 13-in White-Westinghouse TV/VCR was placed directly to the left of the computer and its controls were covered. The TV/VCR was started and stopped by the experimenter. The subjects wore headphones throughout the experiment that were plugged into the TV/VCR. During some phases, the subjects had the opportunity to watch and listen to episodes of one of several popular sitcoms. Subjects chose which sitcom they watched from a list of six. When a video was not being played, static was played on the screen and white noise was played through the headphones.

\section{Experimental Design}

Sessions were 18-min except during shaping, which ended when subjects fulfilled the designated point requirement. Subjects attended two sessions per day for 4 or 5 days per week. A 5 -min break was given for all subjects between sessions.

Six subjects completed the eight phases of the experiment shown in Table 1. All subjects were first exposed to a disruption assessment designed to select subjects whose responding would be disrupted by

\section{Table 1}

Experimental Phases

\author{
Disruption Assessment \\ Shaping \\ Baseline \\ Disruption \\ Instructional Comparison \\ MI Subjects \\ Shaping \\ Baseline \\ Disruption \\ Baseline \\ Disruption
}

Cl Subjects

Instructions

Baseline

Disruption

Baseline

Disruption 
the videos used in the study. These subjects were then matched across two instructional groups on the basis of how much their responding was disrupted. Subjects were grouped depending on whether they received exposure to complete instructions (Cl) or minimum instructions (MI) with the math task. The Ml subjects completed the experiment first and then $\mathrm{Cl}$ subjects were selected. When $\mathrm{Cl}$ subjects were selected, they were matched to the $\mathrm{Ml}$ subjects based on their performance during the disruption assessment.

\section{Procedure}

Disruption assessment. Prior to all sessions, the experimenter greeted subjects and requested their watches. Subjects were asked to sit in front of the computer and follow the instructions on the monitor. All sessions began with the following initial instructions on the computer screen:

Welcome to the human behavior lab! Get comfortable and when you are ready to read the instructions, use the mouse to press the continue button.

The following instructions appeared on screen once the subject pressed the continue button:

Each point earned is exchangeable for 5 cents. Points will be exchanged for money at the end of the experiment. You will be informed of how much you have earned at the end of each day, including your pay for being present for this session. Press the continue button to start the session.

During shaping, subjects learned to perform the dragging task (Aguilera, 2000). Figure 1 shows the stimuli presented during the dragging task. No labels or instructions were included for the subjects, but labels are used here to facilitate the description of the dragging task.

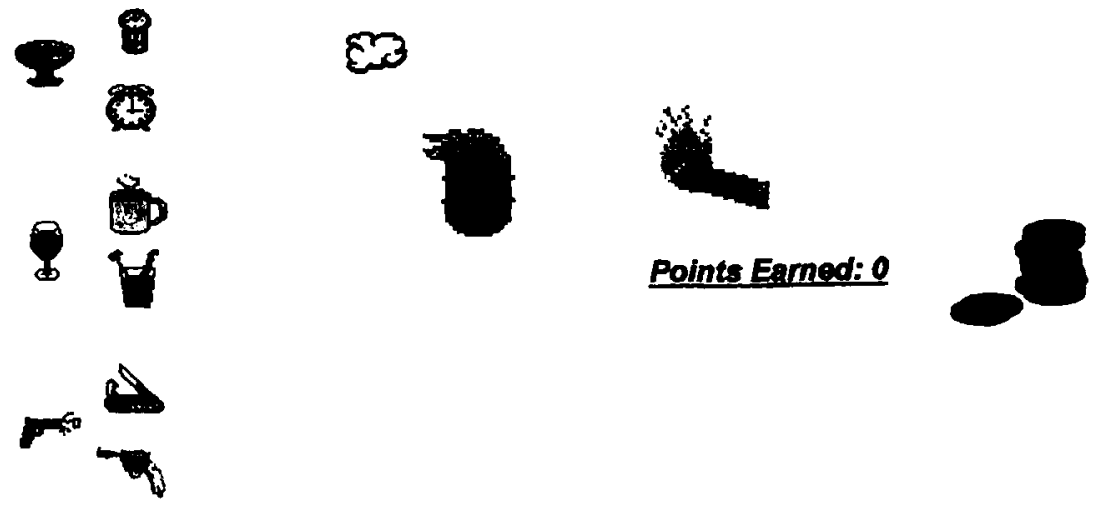

Figure 1. Dragging interface throughout the disruption assessment phase. 
The dragging task consisted of moving three items of a group (e.g., three clocks) into a trash can, lighting the trash can by dragging a match into it, and registering a point earned. Each time a point was registered, a picture of coins appeared on screen for $0.5 \mathrm{~s}$ and the point counter incremented by one. Subjects learned which of the groups of icons led to reinforcement as a conditional discrimination related to the screen color: Clocks were correlated with a blue screen; beverages were correlated with a white screen, and weapons with a yellow screen.

The first object presented on the monitor was the register button (a solid rectangle that appeared on the bottom of the screen). The subject pressed the register button on screen using the cursor and the left mouse button to earn a point. Once the subject registered four points, the trash can and match appeared on screen. The target response was dragging the match icon into the trash can. Each time this response occurred, the trash can lit on fire, a puff of smoke appeared, the register button appeared, and if pressed, a stack of coins appeared and the points counter was incremented. Once the subject had earned two points in a row, the match disappeared and the trash can remained. One of three groups of objects appeared on the screen depending on the background screen color. If the screen color was blue, a group of clocks was presented. If the screen was white, a group of beverages was presented, and if the screen was yellow, a group of weapons was presented. The target response was to drag all three icons of that group into the trash can. Once the target response was reached, the match could be dragged into the trash can, the register button could be pressed, and the consequences occurred. After this sequence of responses was successfully completed five consecutive times, the three groups of objects were presented on screen. The subject could drag only the group of objects paired with the correct background color. The other objects did not move. Once 14 total points were registered, all groups of objects became moveable. If any objects were dragged into the trash can other than the three objects that were conditionally related to the color of the screen, following a drag of the match, the screen reset and subjects did not have the opportunity to earn points.

Shaping of the dragging task continued until the subject registered 40 points. Subjects received these final instructions at the end of each session:

You earned $X$ cents. This session has ended. Please knock on the small door to your right and inform the experimenter that you have finished.

Fourteen subjects did not acquire the dragging response within $18 \mathrm{~min}$ and were debriefed, paid, and dismissed. Those fulfilling this criterion continued with baseline of the disruption assessment phase.

The baseline sessions were identical to the final condition of shaping where the three groups of icons and trash can were displayed. The objects were moveable and differential points were delivered according to the conditional discrimination of screen color and type of object. The stability of response rates was assessed by dividing the final $6 \mathrm{~min}$ of the session 
into two 3-min periods and comparing the mean rate per min of the first 3 $\mathrm{min}$ and the last $3 \mathrm{~min}$ to the overall mean of the final $6 \mathrm{~min}$. Rates were stable when the means of the first $3 \mathrm{~min}$ and the last 3 min were within $10 \%$ of the mean for the final 6 min of the session with no increasing or decreasing trends across the 6-min period. All subjects who completed the experiment reached stability in one baseline session. A session of disruption followed where subjects were asked which of six television situation comedies they preferred to watch. Disruption consisted of videos of the selected show being played on the TVNCR concurrent with the dragging task for a single 18-min session.

The proportion of stable baseline rates during disruption was used to assess response-rate disruption for the dragging task. The proportion of baseline during disruption was calculated by dividing the first, middle, and last $6 \mathrm{~min}$ of disruption by the final 6 stable min of baseline. The proportion of baseline was compared for each block of time within the disruption session to determine differential amounts of disruption throughout that session. The responding of 4 subjects was not disrupted by the videos and they were debriefed, paid, and dismissed. The remaining 6 subjects were divided into the two groups. The disruption ratios of the $\mathrm{Cl}$ subjects during the block of time that was most disrupted were used to match them with $\mathrm{MI}$ subjects with similar disruption ratios. $\mathrm{Cl}$ subjects were matched to MI subjects as closely as possible for disruption ratios and all were within $10 \%$. After the disruption assessment was completed for each subject, that subject moved on to the Instructional Comparison.

Instructional comparison. The instructional conditions were designed to compare the effects of disruption between subjects who received minimum instructions and those who received complete instructions on a new task. A math task was used for both groups. Ml subjects began with shaping to respond to a simple math task. Ml subjects received the initial instructions described earlier with the exception that they were instructed to press the enter key rather than the continue button. Following the initial instructions, a simple addition problem with integers ranging from 0 to 9 appeared on the monitor.

If the Ml subjects typed in the correct answer and pressed the enter key, the computer counted the response as correct. This sequence was measured as one response throughout the experiment. At first, responses were reinforced on a fixed-ratio (FR) 1 schedule of reinforcement. If the answer was correct, an instruction appeared on screen informing the subject to "Press Esc key." Pressing the escape key registered the point. Once the escape key had been pressed, the instruction disappeared, a picture of coins flashed on screen for $0.5 \mathrm{~s}$, one point was recorded on the screen, and a new math problem was generated. If the answer was incorrect and the subject pressed the enter key, a new math problem was generated. Incorrect responses did not count toward fulfilling the ratio. During shaping, Ml subjects were required to complete five FR 1s, five FR $3 s$, and five FR 5s. On the FR $3 s$ and FR 5 s, correct responses that did not fulfill the schedule requirement counted towards fulfilling the ratio and were 
followed by another math problem until the ratio was fulfilled. Points would then be registered as described above. Prior to each component of $5 \mathrm{FRs}$, a prompt came on the screen stating, "Press the enter key to continue."

Once all three FR components were completed, the baseline condition began with the same simple addition problems on a VI 30-s schedule for points. There were $20 \mathrm{VI}$ intervals that ranged from $1 \mathrm{~s}$ to $120 \mathrm{~s}$ (Fleshler \& Hoffman, 1962). All feedback and instructions were the same as they were in shaping. Once an interval timed out, a correct response started a new interval. Subjects responded on the baseline VI 30-s schedule until stability was maintained over a minimum of three sessions. The mean rates of responding for the first session and the last session were required to be within $10 \%$ of the mean rates of the last three sessions combined without an increasing or decreasing trend. Once stability was reached, MI subjects began the disruption condition of the instructional comparison. Subjects selected a video to watch prior to beginning each session of disruption.

$\mathrm{Cl}$ subjects differed from the $\mathrm{Ml}$ subjects in that they received instructions on how to respond to the math task. The instructions began in the instructions condition. First, $\mathrm{Cl}$ subjects received the initial instructions and after pressing the enter key they received the following instruction:

For the following math problems, every correct response will result in 1 point. Press the Enter key to continue.

After pressing enter, the instruction "Every correct response will result in 1 point," appeared and remained on the screen at all times. The second instruction, "Please type in the answer and then press enter," appeared on the screen until a correct answer and enter were pressed. This instruction then disappeared and the "Press ESC key," instruction appeared. A press on the escape key registered the point by increasing the point counter by one. $\mathrm{Cl}$ subjects were required to complete five FR 1 ratios, and then a new instruction appeared: "For the following math problems, every 3 correct responses will result in 1 point. Press the Enter key to continue." The instruction, "Every 3 correct responses will result in 1 point" remained on the screen while the $\mathrm{Cl}$ subjects responded. Correct responses not fulfilling the ratio generated a new math problem. After five FR 3 ratios were completed, this instruction appeared: "For the following math problems, every 5 correct responses will result in 1 point. Press the "Enter" key to continue." The instruction, "Every 5 correct responses will result in 1 point," remained on screen while the $\mathrm{Cl}$ subjects responded.

Following the completion of the instructions condition, the Cl subjects began the baseline condition of the math task. Baseline for $\mathrm{Cl}$ subjects was identical to $\mathrm{Ml}$ subjects except for the difference in instructions. Following the initial instructions, $\mathrm{Cl}$ subjects received the following contingency instructions:

A response will be recorded by pressing the correct sum on the keypad and then pressing the Enter key. Each point must be registered by pressing the Esc key. In order to earn points, you 
should respond approximately once every $X$ seconds. Press the Enter key to begin.

The $X$ in the instruction designated the interresponse time based on the average response rate of the three sessions that met the stability criterion for the yoked MI subject. For instance, if a MI subject responded 36 times per min, the matched $\mathrm{Cl}$ subject was instructed to respond approximately once every $1.6 \mathrm{~s}$. Following these contingency instructions, baseline began. The on-screen instructions during the baseline and disruption conditions for $\mathrm{Cl}$ subjects were identical to those during instructions with the exception that the contingency instruction remained on the screen at all times as well. The remainder of the experimental phases occurred in the same order as was followed for the MI subjects.

\section{Results}

\section{Disruption Assessment}

Table 2 shows $\mathrm{MI}$ and $\mathrm{Cl}$ subjects matched according to disruption of stable baseline drags per min. Disruption ratios were calculated by dividing mean drags per min for the first, middle, and last 6 min of

Table 2

Baseline Response Rates during Disruption Assessment Used to Match $\mathrm{MI}$ and $\mathrm{Cl}$ Subjects and Amount Earned during Instructional Comparison

\begin{tabular}{|c|c|c|c|c|}
\hline \multirow[b]{2}{*}{ Subject } & \multicolumn{3}{|c|}{ Disruption Assessment } & \multirow{2}{*}{$\begin{array}{l}\text { Instructional Comparison } \\
\text { Dollars per hour earned }\end{array}$} \\
\hline & $\begin{array}{l}\text { Baseline } \\
\text { rates }\end{array}$ & $\begin{array}{l}\text { Disruption } \\
\text { rates }\end{array}$ & $\begin{array}{l}\text { Percent of } \\
\text { baseline }\end{array}$ & \\
\hline $\begin{array}{l}\text { Ml-AB } \\
\text { Cl-MC }\end{array}$ & $\begin{array}{r}9.67 \\
10.17\end{array}$ & $\begin{array}{l}8.67 \\
9.50\end{array}$ & $\begin{array}{l}89 \% \\
93 \%\end{array}$ & $\begin{array}{l}5.98 \\
5.64\end{array}$ \\
\hline $\begin{array}{l}\text { MI-SK } \\
\text { CI-NL }\end{array}$ & $\begin{array}{l}7.83 \\
8.00\end{array}$ & $\begin{array}{l}6.83 \\
6.83\end{array}$ & $\begin{array}{l}87 \% \\
85 \%\end{array}$ & $\begin{array}{l}5.67 \\
5.94\end{array}$ \\
\hline $\begin{array}{l}\text { MI-LM } \\
\text { Cl-JC }\end{array}$ & $\begin{array}{r}11.00 \\
7.83\end{array}$ & $\begin{array}{l}8.50 \\
6.83\end{array}$ & $\begin{array}{l}77 \% \\
87 \%\end{array}$ & $\begin{array}{l}5.94 \\
5.97\end{array}$ \\
\hline
\end{tabular}

Note. Percentage of baseline response rates during disruption calculated by dividing mean drags per min during disruption condition by mean drags per min during baseline conditions.

disruption by the drags per min for the final 6 min of stable baseline. The lowest percentage of baseline response rates during disruption was used for matching. All subjects reached stability after one session of baseline during the assessment.

\section{Instructional Comparison}

Table 2 also shows dollars earned per hr for each subject during the instructional comparison. The matched subjects earned approximately the same amount of money across the experiment. Figure 2 shows mean correct responses per min across all sessions of baseline and disruption conditions with the math task. The top, middle, and bottom panels allow 

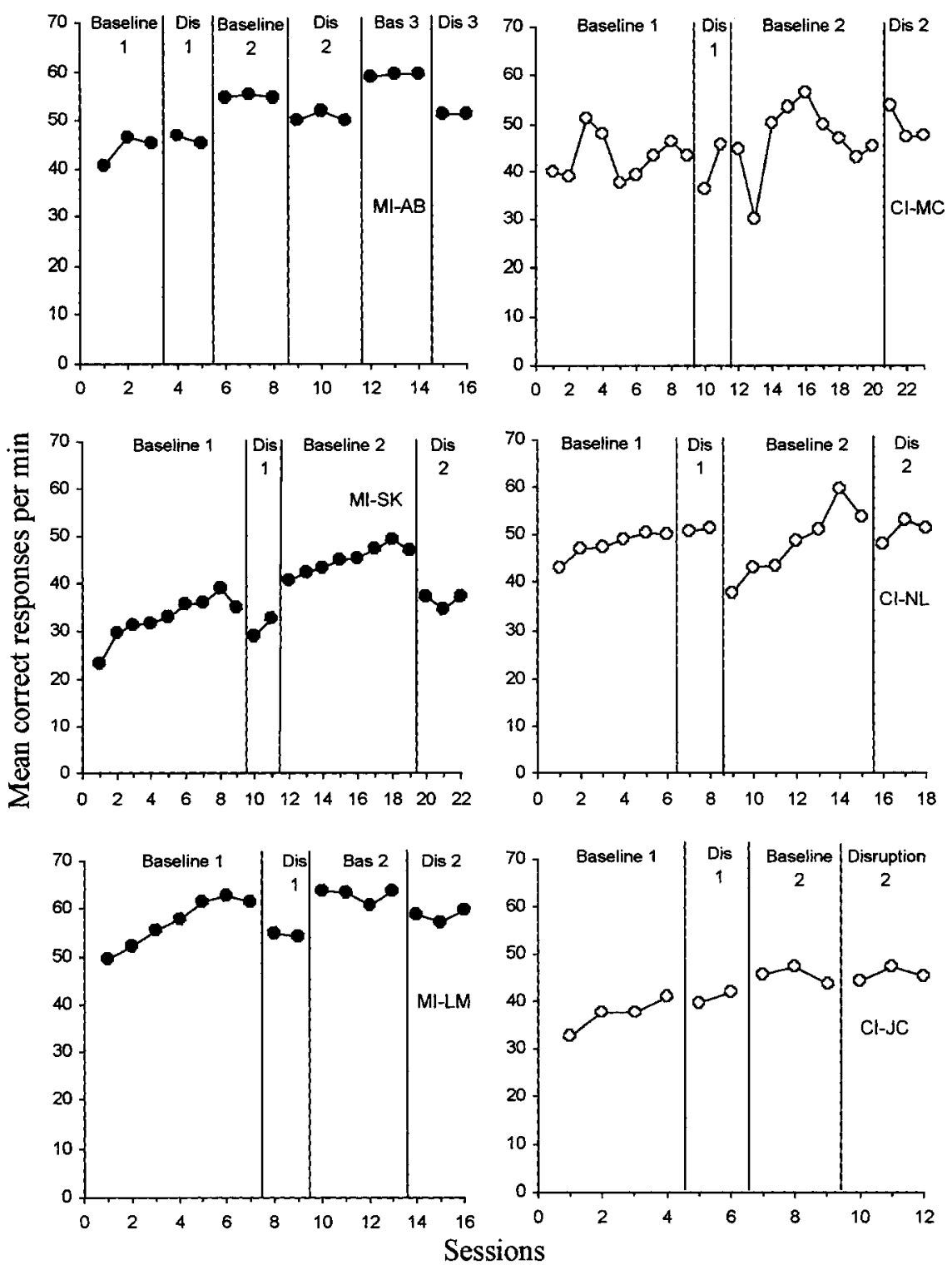

Figure 2. Mean correct responses per minute across sessions of baseline and disruption conditions. Filled circles are for minimally instructed subjects; open circles are for completely instructed subjects.

comparisons between matched subjects. Left panels show the MI subjects and the right panels show the $\mathrm{Cl}$ subjects. Each $\mathrm{Cl}$ subject was instructed to engage in the same rate of behavior as the matched MI subject's stable baseline rate of responding. The top left panel shows that after MI-AB's 
responding reached stability in each baseline, rates remained similar in the first disruption condition, but decreased in both of the subsequent disruption conditions. In addition, MI-AB's rates of correct responding increased each time baseline was introduced. MI-AB was given additional sessions of baseline and disruption in order to determine whether the increase in response rates would continue and it did.

MI-AB's correct rates can be compared to those of Cl-MC in the right top panel of Figure 2. After behavior reached stability in Baseline 1, no systematic rate differences were found in the first disruption condition with decreases in rates occurring in Session 10 but not Session 11. Stable Baseline 2 response rates were similar to the original baseline. The low mean correct response rates in Session 13 correspond to a high number of errors in that session (see Figure 4), not a reduction in rate of responding. Response rates were higher in Disruption 2 than in Baseline 2.

The middle panels of Figure 2 compares matched subjects MI-SK and $\mathrm{Cl}$-NL. MI-SK responding steadily increased across baseline sessions within both conditions until stability was reached. Decreases in response rates occurred in both Disruption 1 and Disruption 2. Stable rates increased from Baseline 1 to Baseline 2. $\mathrm{Cl}-\mathrm{NL}$ responding also increased across baseline sessions until stability was reached. There were no rate decreases in Disruption 1; however, all three sessions of Disruption 2 resulted in a small decrease in response rates from Baseline 2. Stable rates were slightly higher in Baseline 2 compared to Baseline 1.

The bottom panels of Figure 2 compare matched subjects MI-LM and $\mathrm{Cl}-\mathrm{JC}$. There were no differences in stable baseline sessions across the two baseline conditions for MI-LM; however decreases in responding occurred in both Disruption 1 and Disruption 2. $\mathrm{Cl}-\mathrm{JC}$ response rates decreased in the first session of Disruption 1 and increased during the second session. No difference in rates occurred in Disruption 2.

Figure 3 shows the proportion of stable baseline correct responses rates that occurred during disruption sessions for each pair of subjects. Proportions were calculated for all subjects by dividing the mean correct responses per min in disruption sessions by the mean correct responses per min for the preceding three stable baseline sessions. The top panel shows that in both sessions of Disruption 1, MI-AB's response rates increased compared to baseline. MI-AB's responding was disrupted across all three sessions of Disruption 2 and in both sessions of Disruption 3. Cl-MC's responding decreased in the first session of Disruption 1, but increased during the second session (no disruption effect). In Disruption 2 , proportions for $\mathrm{Cl}-\mathrm{MC}$ show rates greater than baseline across all three sessions and no disruption effect. Cl-MC did not receive a third manipulation of baseline and disruption.

The middle panel of Figure 3 shows the same proportions for matched subjects MI-SK and CI-NL. Subject MI-SK had a greater proportion of disruption across both Disruption 1 and Disruption 2 compared to $\mathrm{Cl}-\mathrm{NL}$. For MI-SK, the decrease in responding during disruption was substantial in four out of five disruption sessions. $\mathrm{Cl}-\mathrm{NL}$ showed no disruption during 

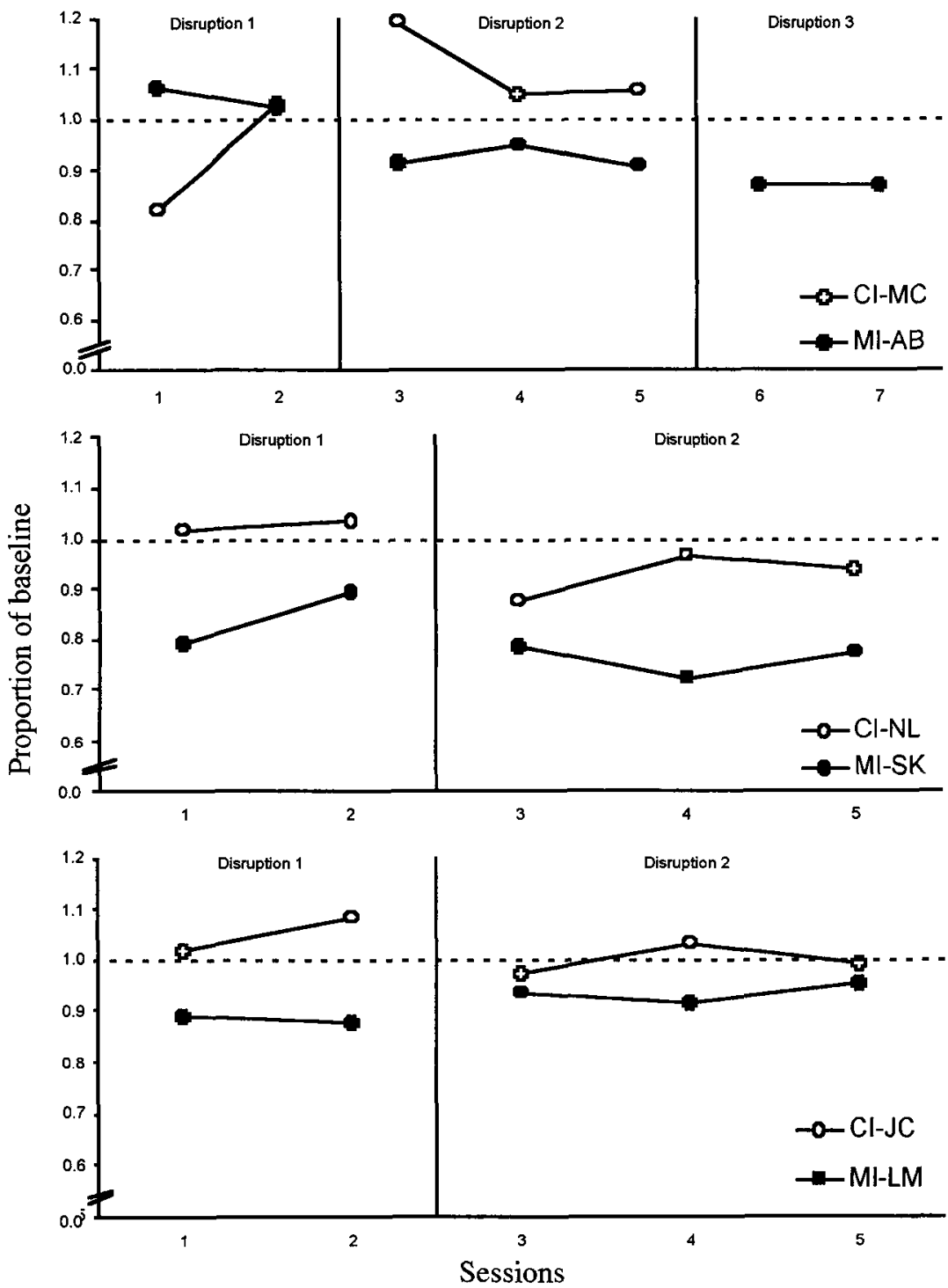

Figure 3. Proportion of stable baseline correct response rates that occurred during disruption sessions for each pair of subjects. Proportions calculated by dividing the correct responses per min in disruption phases by the mean correct responses per min for the preceding three stable baseline sessions. Horizontal line from 1.0 indicates baseline rates. Filled circles are for minimally instructed subjects; open circles are for completely instructed subjects.

Disruption 1, and though disruption occurred during all sessions of Disruption 2, the proportion of baseline was always higher than MI-SK. For both subjects, disruption increased from Disruption 1 to Disruption 2. 
The bottom panel of Figure 3 shows the same proportions for matched subjects $\mathrm{MI}-\mathrm{LM}$ and $\mathrm{Cl}-\mathrm{JC}$. $\mathrm{Cl}-\mathrm{JC}$ responding was less disrupted than MI-LM responding across both disruption conditions. MI-LM rates were disrupted in all sessions of Disruption 1 and Disruption 2, with less disruption in Disruption 2. Cl-JC response rates were not disrupted in 4 out of 5 disruption sessions. In all sessions there was less disruption for Cl-JC than MI-LM.
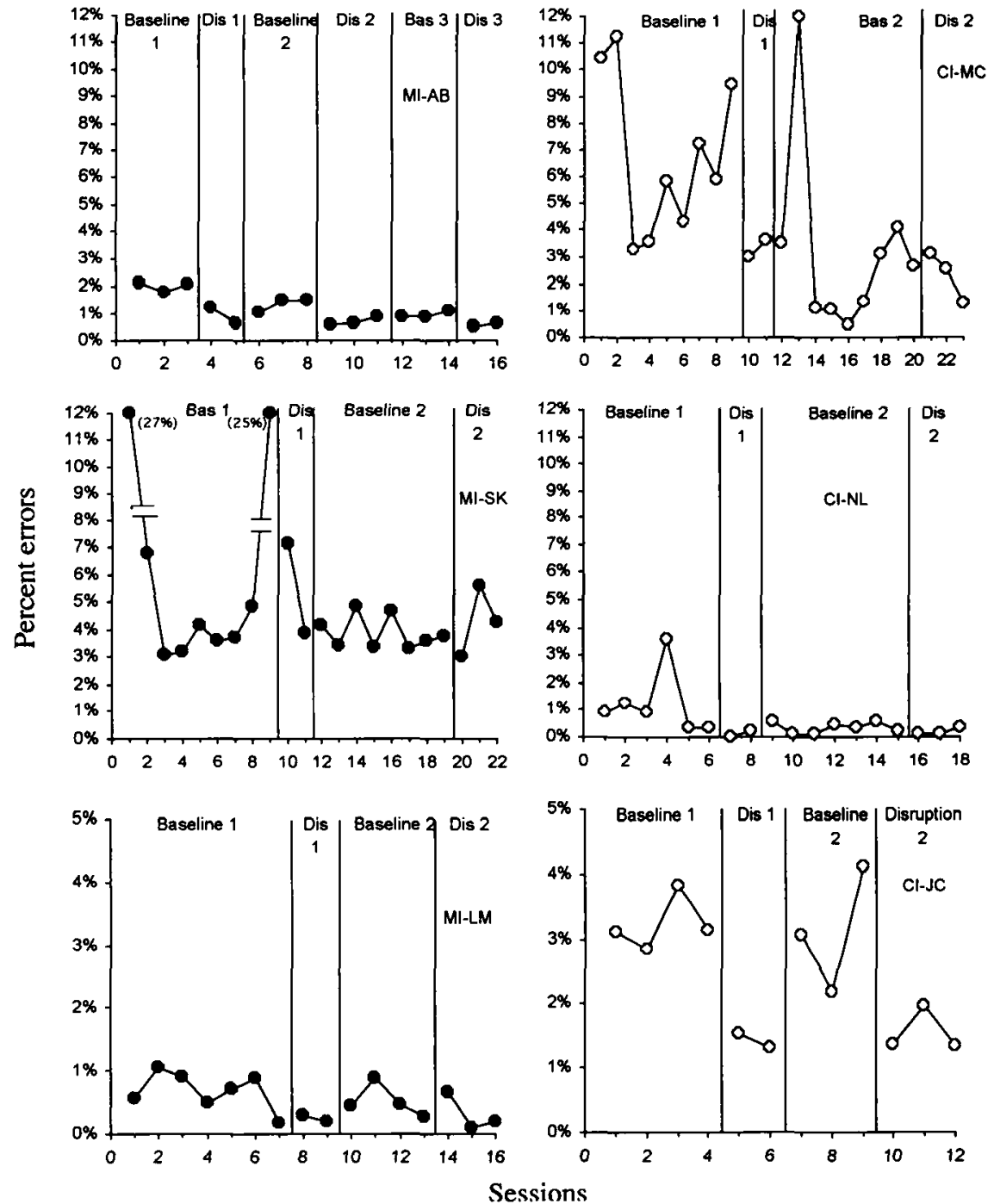

Figure 4. Percentages of errors per session over baseline and disruption phases. Filled circles are for minimally instructed subjects; open circles are for completely instructed subjects. 
Figure 4 shows the percentages of errors per session for each pair of subjects. Percentages were calculated for all subjects by dividing the total number of errors per session by the sum of total correct responses and total errors per session. The top panel shows a decreasing trend across conditions for $\mathrm{MI}-\mathrm{AB}$ and $\mathrm{Cl}-\mathrm{MC}$. For $\mathrm{MI}-\mathrm{AB}$, the percentage of errors was smaller in each disruption condition compared to baseline conditions whereas the percentage of errors decreased clearly only in Disruption 1 for $\mathrm{Cl}-\mathrm{MC}$. Cl-MC had a greater percentage of errors across all conditions than MI-AB. For $\mathrm{Cl}-\mathrm{MC}$, there was a larger number of errors per session in Baseline 1 than in other conditions. Other than in Session 13 , the proportion of errors per session was similar across the remaining three conditions.

The middle panel of Figure 4 compares errors for matched subjects MI-SK and $\mathrm{Cl}-\mathrm{NL}$ over baseline and disruption conditions. In the top panel, the percentage of errors for MI-SK shows no differences across baseline and disruption conditions other than the outliers in Sessions $1(27 \%)$ and $9(25 \%)$ in Baseline 1. Compared to Cl-NL in the bottom panel, MI-SK showed a greater percentage of errors across each condition. Cl-NL's percentage of errors showed slight decreases in disruption conditions.

The bottom panel of Figure 4 compares errors for matched subjects MI-LM and Cl-JC. A decrease in the percentage of errors per session was found in both disruption conditions for $\mathrm{Cl}-\mathrm{JC}$, however, this effect is not clear for MI-LM. CI-JC had a greater percentage of errors per session than MI-LM across all conditions and had greater decreases in the percentage of errors per session in both disruption conditions.

The proportion of stable baseline response rates that occurred during disruption was calculated with errors included (overall response rates); however, these data are not included because the differences between the $\mathrm{MI}$ and $\mathrm{Cl}$ subjects were redundant with those shown in Figure 3.

\section{Discussion}

The results demonstrate that in 14 out of 15 disruption sessions for which the performance of $\mathrm{Ml}$ and $\mathrm{Cl}$ subjects can be compared, the proportions of baseline correct response rates were greater for $\mathrm{Cl}$ subjects than $\mathrm{MI}$ subjects. The only exception occurred on the first session of Disruption 1 for $\mathrm{MI}-\mathrm{AB}$ and $\mathrm{Cl}-\mathrm{MC}$. This differential disruption effect occurred independent of the percentage of errors across baseline and disruption conditions. Overall, the data indicate that $\mathrm{Cl}$ subjects' performance was more resistant to change than $\mathrm{Ml}$ subjects for subjects whose responding was disrupted by videos of popular television situation comedies.

These results are consistent with the hypothesis that rule-governed behavior can be stronger or more resistant to change than specific classes of contingency-governed behavior. Because other variables that have been shown to affect resistance to change (e.g., rate of reinforcement) were held constant in the current experiment, it is assumed the differential performance in the presence of the disrupter was due to the instructions 
provided and the extensive rich reinforcement history for following such rules for the subjects.

It is not difficult to assume that following rules like those used in the current experiment would result in greater resistance to change. In most humans' reinforcement histories, the proportion of behaviors that are reinforced, both through positive and negative reinforcement, is likely to be high in the presence of rules and instructions that correspond to local contingencies. Reinforcement for instruction following in such cases occurs from both the verbal community (e.g., parents, siblings, and teachers) and from the nonsocial contingencies that correspond with the instructions (i.e., instructions that work). This history can be contrasted with the brief history of performing math problems for points on a VI-30 schedule used in the current experiment. The MI subjects likely have a history with only the latter contingencies within the present experiment. These differences in reinforcement could then be responsible for the results in the present experiment where $\mathrm{Cl}$ subjects' behavior was more resistant to change than $\mathrm{MI}$ subjects' behavior when the only difference was the presence and absence of instruction.

Further, differences in the history of reinforcement for rule-governed behavior may account for differences in sensitivity found across individuals. This is consistent with the account of rule following suggested by Wulfert et al. (1994). They found that subjects scoring both high and low on a personal rigidity scale performed in accordance with changes in instructions when those instructions accurately described the contingencies. Subjects scoring higher on the rigidity scale, however, were more likely to continue to respond in accordance with instructions when those instructions became inaccurate than were subjects scoring lower on the rigidity scale. Such rigidity was suggested to be a product of a response class primarily involving a history of compliance with instructions.

\section{Further Research on Reinforcement Histories}

Overall the results suggest that rule-governed behavior manipulated in the current experiment is more resistant to change than contingencygoverned behavior. In order to determine whether the same reinforcement principles govern sensitivity and resistance to change, however, requires further experiments. The current research did not change the schedule of reinforcement to test whether rule-governed behavior and contingency-governed behavior were differentially sensitive. In addition, rate of reinforcement was not manipulated. For example, if the rate of reinforcement for MI subjects' performance were richer, perhaps the performance of the Ml subjects would also be more resistant to the disrupter. Alternatively, if the rate of reinforcement for the performance of the $\mathrm{Cl}$ subjects was decreased, then their resistance should decrease.

Examining the effects of a history of reinforcement for rule following on resistance to change might extend the generality of the relation between sensitivity and resistance to change and further the understanding of the principles governing instructional control. To conduct such studies it might 
be necessary to include conditions in which both traditional measures of sensitivity and resistance to change are tested. For example, studies have shown that instructed FR performance is likely to be insensitive to FI schedules of reinforcement, whereas instructing both FR and DRL performance is likely to lead to sensitivity to $\mathrm{FI}$ schedules (LeFrancois et al., 1988; Torgrud \& Holborn, 1990). These studies have used a test procedure in which the instructed schedule is changed to the FI schedule without instructing the subjects. If the subjects' behavior changes then it is considered sensitive to the FI schedule. Replicating this effect within studies that also involve disruption conditions would allow measuring resistance to change and sensitivity and then better evidence that sensitivity and strength are similar phenomena would be obtained.

In addition to these kinds of future investigations, disruption may be increased if the reinforcer magnitude for watching the video was greater. Responding to the schedule of reinforcement and the video disrupter will reflect the amount of reinforcement from these sources. If there is an alternative source of reinforcement available, responding should match the two available alternatives for responding based on the amount of reinforcement of those two alternatives (McDowell, 1988). This could be achieved in future research by having all subjects answer questions about the television shows they have watched and to award points exchangeable for money for the answers. If watching the television shows results in more reinforcers, then a greater separation between $\mathrm{MI}$ subjects and $\mathrm{Cl}$ subjects might be found.

\section{Strength and Instructed Variability}

Although the interest in the current experiment was the relation between insensitivity and resistance to change when behavior is under instructional control, instructions do not always produce insensitivity. Instructed behavior can be made sensitive to the changes in local contingencies of reinforcement usually through the production of behavior that is variable enough during changes in contingencies such that behavior contacts these changes and becomes controlled by them (Galizio, 1979; Joyce \& Chase, 1990; LeFrancois et al., 1988). Further, Bicard and Neef (2002) and Joyce and Chase (1990) have showed that strategic instructions are likely to produce variability in behavior that helps produce sensitivity. Of future interest is the relation between different kinds of instructions, sensitivity, and resistance to change. The current experiment opens the door for a program of research that identifies the kinds of instructions that increase or decrease resistance to change.

\section{Error Analysis}

One of the more curious findings of the experiment was the decrease in errors produced during the disruption condition. Some of these decrements were hardly discernible, for example those between Baseline 1 and Disruption 1 for $\mathrm{MI}-\mathrm{AB}$ and $\mathrm{Cl}-\mathrm{NL}$. Others however were quite pronounced, for example, both switches for subject $\mathrm{Cl}-\mathrm{JC}$. 
These reductions did not appear to be related to whether or not subjects experienced completed or minimal instructions, and perhaps they are due to uncontrolled variability, but they did occur in 10 of the 13 switches between baseline and disruption conditions. The interest in errors, then, goes beyond questions of instructional control to examining further the effects of disruption on the accuracy of responding. Determining the relation between errors and disruption under various conditions of reinforcement rate needs to be investigated further (Dube \& Mcllvane, 2001).

\section{Conclusion}

Behavior that was instructed was found to be more resistant to change than behavior that was governed by contingencies. Because the contingencies for rule following have a rich history for most humans, the present study suggests that this extensive history has similar effects on resistance to change as it does on sensitivity to changing contingencies. Further analyses of the relation between instructions and resistance to change have powerful implications for educational and applied settings. Understanding the relation between sensitivity and strength under these conditions may improve our ability to understand how to predict and control performances under instructional control. In addition, resistance to change may provide an additional test for differences between instructed and contingency-governed behavior.

\section{References}

AGUILERA, C. (2000). Effects of reinforcement history for following rules on sensitivity to contingencies of reinforcement. Unpublished master's thesis, West Virginia University, [On-line abstract]. Available: http://etd.wvu.edu/ templates/showETD.cfm? recrum $=1764$

BICARD, D. F., \& NEEF, N. A. (2002). Effects of strategic versus tactical instructions on adaptation to changing contingencies in children with ADHD. Journal of Applied Behavior Analysis, 35, 375-389.

DUBE, W. V., \& MCILVANE, W. J. (2001). Behavioral momentum in computerpresented discriminations in individuals with severe mental retardation. Journal of the Experimental Analysis of Behavior, 75, 15-23.

FLESHLER, M., \& HOFFMAN, H. S. (1962). A progression for generating variableinterval schedules. Journal of the Experimental Analysis of Behavior, 5(4), 529-530.

GALIZIO, M. (1979). Contingency-shaped and rule-governed behavior: Instructional control of human loss avoidance. Journal of the Experimental Analysis of Behavior, 31, 45-49.

JOYCE, J. H., \& CHASE, P. N. (1990). Effects of response variability on the sensitivity of rule-governed behavior. Journal of the Experimental Analysis of Behavior, 54, 251-262.

KAUFMAN, A., BARON, A., \& KOPP, R. E. (1966). Some effects of instructions on human operant behavior. Psychonomic Monograph Supplements, 1, 243-250. 
LEFRANCOIS, J. R., CHASE, P. N., \& JOYCE, J. H. (1988). The effects of a variety of instructions on human fixed-interval performance. Journal of the Experimental Analysis of Behavior, 49, 383-393.

MACE, F. C., LALLI, J. S., SHEA, M. C., LALLI, E. P., WEST, B. J., ROBERTS, M., \& NEVIN, J. A. (1990). The momentum of behavior in a natural setting. Journal of the Experimental Analysis of Behavior, 54, 163-172.

MCDOWELL, J. J (1988). Matching theory in natural human environments. The Behavior Analyst, 11(2), 95-109.

NEVIN, J. A., MANDELL, C., \& ATAK, J. R. (1983). The analysis of behavioral momentum. Journal of the Experimental Analysis of Behavior, 39, 49-59.

SHIMOFF, E., CATANIA, A. C., \& MATTHEWS, B. A. (1981). Uninstructed human responding: Sensitivity of low-rate performance to schedule contingencies. Journal of the Experimental Analysis of Behavior, 36, 207-220.

SKINNER, B. F. (1969). Contingencies of reinforcement: A theoretical analysis. Englewood Cliffs, NJ: Prentice-Hall.

TORGRUD, L. J., \& HOLBORN, S. W. (1990). The effects of verbal performance descriptions on nonverbal operant responding. Journal of the Experimental Analysis of Behavior, 54(3), 273-291.

WULFERT, E., GREENWAY, D. E., FARKAS, P., HAYES, S. C., \& DOUGHER, M. J. (1994). Correlation between self-reported rigidity and rule-governed insensitivity to operant contingencies. Journal of Applied Behavior Analysis, $27,659-671$. 